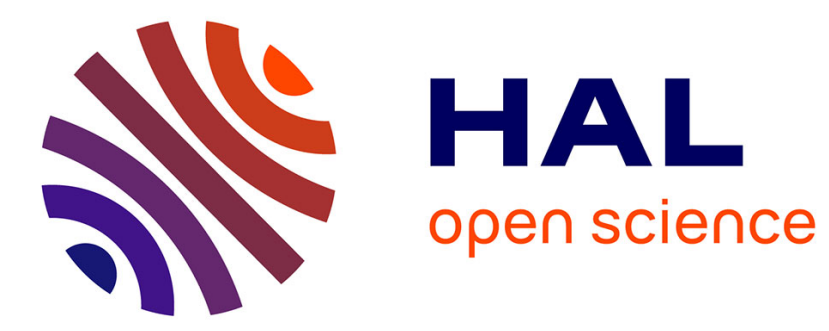

\title{
Classification of SD-OCT Volumes for DME Detection: An Anomaly Detection Approach
}

Shrinivasan Sankar, Désiré Sidibé, Carol Y. Cheung, Tien Y. Wong, Ecosse Lamoureux, Dan Milea, Fabrice Mériaudeau

\section{- To cite this version:}

Shrinivasan Sankar, Désiré Sidibé, Carol Y. Cheung, Tien Y. Wong, Ecosse Lamoureux, et al.. Classification of SD-OCT Volumes for DME Detection: An Anomaly Detection Approach. SPIE Medical Imaging, Feb 2016, San Diego, United States. hal-01390683

\section{HAL Id: hal-01390683 \\ https://u-bourgogne.hal.science/hal-01390683}

Submitted on 2 Nov 2016

HAL is a multi-disciplinary open access archive for the deposit and dissemination of scientific research documents, whether they are published or not. The documents may come from teaching and research institutions in France or abroad, or from public or private research centers.
L'archive ouverte pluridisciplinaire HAL, est destinée au dépôt et à la diffusion de documents scientifiques de niveau recherche, publiés ou non, émanant des établissements d'enseignement et de recherche français ou étrangers, des laboratoires publics ou privés. 


\title{
Classification of SD-OCT Volumes for DME Detection: An Anomaly Detection Approach
}

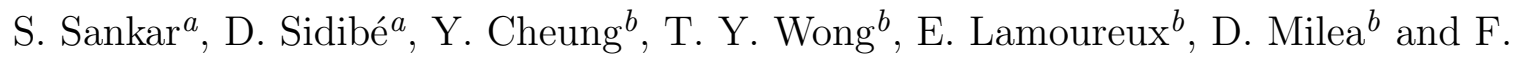 \\ Mériaudeau $^{a}$ \\ ${ }^{a}$ LE2I UMR6306, CNRS, Arts et Mtiers, Univ. Bourgogne Franche-Comt. \\ ${ }^{b}$ Singapore Eye Research Institute, Singapore National Eye Center, Singapore.
}

\begin{abstract}
Diabetic Macular Edema (DME) is the leading cause of blindness amongst diabetic patients worldwide. It is characterized by accumulation of water molecules in the macula leading to swelling. Early detection of the disease helps prevent further loss of vision. Naturally, automated detection of DME from Optical Coherence Tomography (OCT) volumes plays a key role. To this end, a pipeline for detecting DME diseases in OCT volumes is proposed in this paper. The method is based on anomaly detection using Gaussian Mixture Model (GMM). It starts with pre-processing the B-scans by resizing, flattening, filtering and extracting features from them. Both intensity and Local Binary Pattern (LBP) features are considered. The dimensionality of the extracted features is reduced using PCA. As the last stage, a GMM is fitted with features from normal volumes. During testing, features extracted from the test volume are evaluated with the fitted model for anomaly and classification is made based on the number of B-scans detected as outliers. The proposed method is tested on two OCT datasets and achieved a sensitivity and a specificity of $80 \%$ and $93 \%$ on the first dataset, and $100 \%$ and $80 \%$ on the second one. Moreover, the experiments show that the proposed method achieves better classification performances than other recently published works.
\end{abstract}

Keywords: Diabetic Macular Edema, SD-OCT, Gaussian Mixture Model, Local Binary Pattern.

\section{INTRODUCTION}

Hyperglycemia is a well known condition caused by increased glucose level in blood, commonly known as Diabetes. Hyperglucemia damages blood vessels. Any complications arising due to damage caused to the retina by Hyperclycemia is dubbed Diabetic Retinopathy (DR). A common type of DR in type 2 diabetic patients is Diabetic Macular Edema (DME) which is caused by accumulation of fluid in the broken down blood-retinal barrier. DME is the primary cause of blindness in diabetic patients. Early detection of DME can prevent blindness leading only to slight deterioration of vision.

Despite this fact, there is need to rely on the availability of skilled clinicians to evaluate images of the retina to diagnose the disease. Fully automated methods using Computer Aided Diagnosis (CAD) systems to identify the disease at an early stage can play a crucial role in disease detection. Though there are two imaging modalities (fundus photography and spectral domain OCT (SD-OCT)) currently available to image the retina, challenges manifest themselves in both of them. In case of OCT images, the size, shape and location of the disease features in the OCT volumes make it difficult to classify them as diseased.

With a view to automate the detection of DME, we propose a novel method using recent image processing techniques coupled with machine learning approaches that need minimal parameters during the training process. Given that OCT images are volumetric data, we also propose a way to localise the disease in the entire volume by automatically detecting individual B-scans as diseased. The rest of the paper consists of a section to detail the methodlogy of our approach followed by the experiments and results section.

Further author information: (Send correspondence to D. Sidibé)

D. Sidibé: E-mail: dro-desire.sidibe@u-bourgogne.fr 


\section{METHODOLOGY}

The proposed method is based on an anomaly detection approach using Gaussian Mixture Model (GMM). The method can be broadly divided into pre-processing, GMM modeling and abnormal B-scans detection.

\subsection{Pre-processing}

The SD-OCT data is organized as a 3D cube, i.e. a series of slices called B-scans, and each B-scan is a crosssectional image of the retina. Given that the size of the B-scans in the datasets are large, we started our pre-processing by resizing each B-scan to a fixed size of $400 \times 512$. Then, we applied an image denoising method to reduce the speckle noise in each B-scan, since SD-OCT images are known to be corrupted by a speckle noise. ${ }^{2}$ In our work, we used the Non-Local Means (NL-means) algorithm ${ }^{3}$ which offers the advantage to reduce the noise while preserving the details and texture of the original image. Each B-scan is then flattened following the procedure proposed described in, ${ }^{4}$ to ensure that the dominant features, in each B-scan, are aligned in a nearly horizontal line. Finally, features are extracted from each B-scan. In this work, both intensity and texture are considered. We used LBP features to describe OCT images texture. The feature vector corresponding to each B-scan in a SD-OCT volume are stacked as columns of a matrix which represent the volume.

\subsection{GMM Modeling}

We consider that we have a set of $N$ SD-OCT volumes which are known to be from healthy patients. We want to use this data to model the global appearance of normal SD-OCT images using a GMM. We first represent each B-scan as a vector $b$ of size $d=W H$ obtained by concatenation of the rows of the $W \times H$ 2D image. Then, we can represent the whole SD-OCT volume as a set of $N^{\prime}$ vectors in $\mathbb{R}^{d}$ each corresponding to one B-scan of the volume: $V=\left\{b_{1}, b_{2}, \ldots, b_{N^{\prime}}\right\}, b_{i} \in \mathbb{R}^{d}$ for all $i=1, \ldots, N^{\prime}$, and $N^{\prime}$ is the number of B-scan in each volume.

If we put together all B-scans from the $N$ training normal SD-OCT volumes, we create a large data matrix $\mathbf{X}$ whose columns are the B-scans from all the volumes: $\mathbf{X}=\left[b_{1}, b_{2}, \ldots, b_{M}\right]$, with $M=N N^{\prime}$ the total number of normal B-scans.

Note that since each B-scan is a vector in a $d$-dimensional space, with $d=W H$, we have a very high dimensional feature space. For example, if each B-scan is a $400 \times 512$ image, then $d=204800$. Therefore, we apply principal components analysis $(\mathrm{PCA})$ to reduce the dimensionality of the data. We first normalize the data by removing the mean vector, and we compute the covariance matrix of the data as $\mathbf{C}=\frac{1}{M} \mathbf{X X}^{T}$. The eigenvectors of $\mathbf{C}$ form the set of principal components of the data. The corresponding eigenvalues $\lambda_{i}$, $i=1, \ldots, d$, give the importance of each axis. For dimensionality reduction, we keep the first $p$ dominant principal components corresponding to the $p$ largest eigenvalues. We set the value of $p$ such that at least $95 \%$ of the total variance of the data is retained. So, we set $p$ such that $\sum_{i=1}^{p} \lambda_{i} / \sum_{i=1}^{d} \lambda_{i} \geq 0.95$. PCA typically reduces the dimension of the data from $d=204800$ to few hundreds, i.e. $p=300$ or $p=500$ as will be shown in Section 3 .

In the reduced $p$-dimensional space, we represent the distribution of normal B-scans' appearance with a Gaussian mixture model (GMM). A GMM is a parametric probability density function represented as a weighted sum of $K$ Gaussian component densities: ${ }^{5}$

$$
p(\mathbf{x} \mid \theta)=\sum_{i=1}^{K} w_{i} g_{i}\left(\mathbf{x} \mid \mu_{i}, \Sigma_{i}\right)
$$

where $\mathbf{x} \in \mathbb{R}^{p}, w_{i}, i=1, \ldots, K$ are the mixture weights which satisfy $\sum_{i=1}^{K} w_{i}=1$, and $g_{i}\left(\mathbf{x} \mid \mu_{i}, \Sigma_{i}\right)$ is the $i$-th component of the mixture model. Each component of the model is a $p$-dimensional multivariate Gaussian defined by:

$$
g_{i}\left(\mathbf{x} \mid \mu_{i}, \Sigma_{i}\right)=\frac{1}{(2 \pi)^{p / 2}\left|\Sigma_{i}\right|^{1 / 2}} \exp \left\{-\frac{1}{2}\left(\mathbf{x}-\mu_{i}\right)^{T} \Sigma_{i}^{-1}\left(\mathbf{x}-\mu_{i}\right)\right\}
$$

where $\mu_{i} \in \mathbb{R}^{p}$ is the mean vector of the Gaussian, and $\Sigma_{i}$ is the $p \times p$ covariance matrix.

The parameters of the model $\theta=\left\{w_{i}, \mu_{i}, \Sigma_{i}, i=1, \ldots, K\right\}$, are learned from the set of training data using the expectation-maximization (EM) algorithm which iteratively estimates the parameters of the model. ${ }^{5}$

A flowchart of the method for representing the appearance of normal B-scans as a GMM is shown in Fig. 1. 


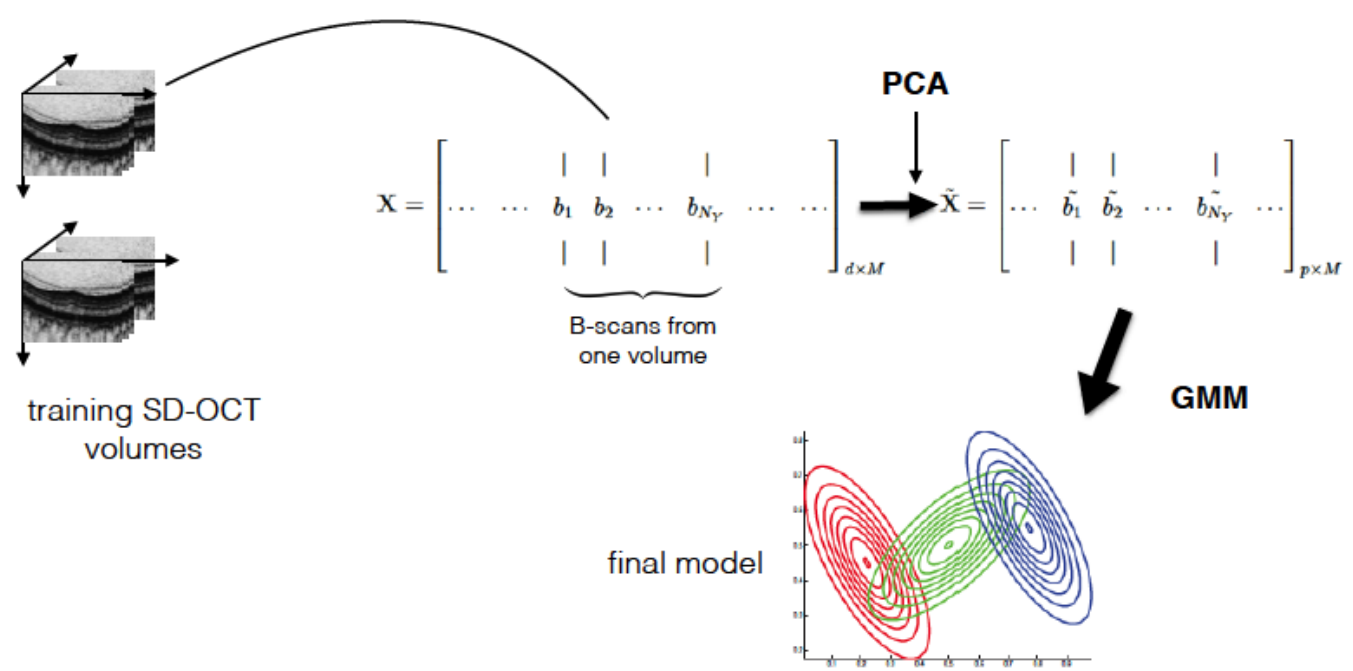

Figure 1: Flowchart of the Gaussian mixtures model creation.

\subsection{Abnormal B-scans Detection}

Our method is based on the identification of abnormal B-scans, i.e. B-scans showing visible signs of retinal diseases, using the obatined GMM from previous section. Given a B-scan $\mathbf{b}$ of size $W \times H$, we first represent it as a vector in $\mathbb{R}^{d}, d=W H$, and project this $d$-dimensional vector onto the set of principal components found in Section 2.2, to obtain $\tilde{\mathbf{b}} \in \mathbb{R}^{p}$, where $p \ll d$.

Then, we compute the Mahalanobis distance from $\tilde{\mathbf{b}}$ to the GMM as:

$$
\Delta_{G M M}(\tilde{\mathbf{b}})=\underset{i}{\operatorname{argmin}} \Delta_{i}(\tilde{\mathbf{b}})
$$

where $\Delta_{i}(\tilde{\mathbf{b}})$ is the distance from $\tilde{\mathbf{b}}$ to the $i$-th component of the GMM defined by:

$$
\Delta_{i}(\tilde{\mathbf{b}})=\left(\tilde{\mathbf{b}}-\mu_{i}\right)^{T} \Sigma_{i}^{-1}\left(\tilde{\mathbf{b}}-\mu_{i}\right) .
$$

Finally, we consider as abnormal, B-scans with distances from the GMM above a certain threshold value. To explain how we find this threshold value, let us first consider the case of $1 \mathrm{D}$ Gaussian distributions. If $\mathbf{x}$ is a $1 \mathrm{D}$ random variable normally distributed as $\mathbf{x} \sim \mathcal{N}(\mu, \sigma)$, then $P(|\mathbf{x}-\mu| \leq 2 \sigma)=0.95$. Which means that $\mathbf{x}$ does not agree with the Gaussian model if $|\mathbf{x}-\mu|>2 \sigma$, and $\mathbf{x}$ is therefore considered as an anomaly or outlier. This is the well known $2 \sigma$-rule for $1 \mathrm{D}$ normal distributions.

This idea can be extended to the case of multivariate $p$-dimensional Gaussian distributions. First, it can be shown that the Mahalanobis distance defined in Equation (4) follows a $\chi^{2}$-distribution with $p$ degrees of freedom, since it is the sum of $p$ Gaussian random variables: ${ }^{5} \Delta_{G M M} \sim \chi_{p}^{2}$. We can then detect outliers as data points in $\mathcal{R}^{p}$ with a Mahalanobis distance that does not fit the $\chi_{p}^{2}$-distribution. We set the threshold value to be equal to the $97.5 \%$ quantile of $\chi^{2}$-distribution with $p$ degree of freedom, i.e. $\delta=\chi_{p: 0.975}^{2}$, and we classify a B-scan $\mathbf{b}$ using the following rule:

$$
\mathbf{b} \quad \text { is abnormal if } \quad \Delta_{G M M}(\tilde{\mathbf{b}})>\delta \text {. }
$$

Figure 2 summarizes the abnormal B-scans detection procedure using a GMM.

The final step of the method is the identification of patients with retinal diseases, such as DME, versus normal subjects. An SD-OCT volume is classified as normal or abnormal based on the number of detected abnormal B-scans. Ideally, a normal OCT volume should contain no abnormal B-scan, while an abnormal one should contain many. 

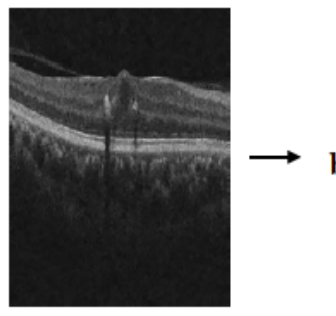

B-scan

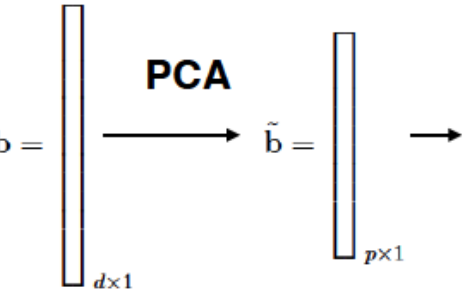

dimension reduction

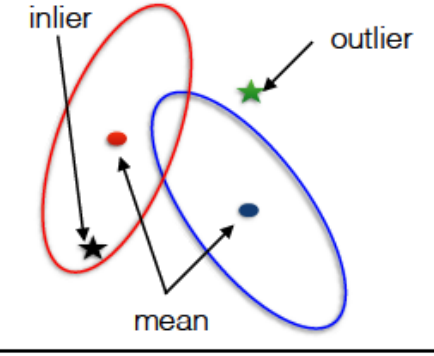

GMM

Figure 2: Flowchart of the Gaussian mixtures model creation.

\section{EXPERIMENTS AND RESULTS}

The proposed framework was tested on two different datasets acquired by different groups, in different places using different SD-OCT cameras:

- The first dataset, SERI, consists of 32 SD-OCT volumes (16 DME and 16 normal cases), and is provided by Singapore Eye Research Institute (SERI). This data is acquired using CIRRUS TM (Carl Zeiss Meditec, Inc., Dublin, CA) SD-OCT camera, and each OCT volume contains 128 B-scans each of size $400 \times 512$ pixels. All 32 volumes were read and assessed by trained graders and classified as normal or DME based on the presence specific signs such as retinal thickening, hard exudates, and intra-retinal cystoid space.

- The second dataset, which we refer to as Duke, is provided by Duke University. ${ }^{6}$ The dataset consists in 30 SD-OCT volumes from 15 DME patients and 15 normal subjects, respectively. The data is acquired using Spectralis (Heidelberg Inc., Heidelberg, Germany) camera.

\subsection{Training and Model selection}

One of the key parameters of the method is the number $K$ of Gaussians used in the GMM. For a given dataset, we use part of the normal SD-OCT data for training, i.e. for learning the GMM. For SERI dataset, we use 11 of the 16 normal volumes for training. More precisely, we use 8 of the 11 training volumes for fitting a GMM with $K$ components, and we use the remaining 3 volumes for validation. This is done by checking each B-scan of the validation OCT volumes against the learned GMM, and detecting outliers using Equation 5. For each value of $K$, we repeat the cross-validation experiment, i.e. training with 8 volumes and testing with 3,10 times and report the average classification accuracy.

A similar training procedure is used for the Duke dataset. However, since this dataset contains 15 normal patients, we use 10 normal SD-OCT volumes to learn the GMM.

The average number of detected outliers obtained for different $K$ values are shown in Table 1 . As can be seen, for Duke dataset the best classification acuracy is obtained with $K=8$ independently of the feature used. For SERI dataset, the best model is obtaines with $K=8$ for intensity features, and with $K=12$ with LBP features.

In both cases, after chosing the best value for $K$ by cross-validation, the final GMM model is fitted with all the training OCT volumes.

\subsection{Testing}

After model selection, the final model is tested with the remaining control volumes (5 for both SERI and Duke datasets) and all of the DME volumes (16 in SERI and 15 in Duke dataset). 
Table 1: Average number of detected outliers for different $K$ in fitting the GMM.

\begin{tabular}{|c|c|c|c|c|}
\hline \multirow{2}{*}{ GMM $N$} & \multicolumn{2}{|c|}{ SERI } & \multicolumn{2}{c|}{ DUKE } \\
\cline { 2 - 5 } & Intensity & LBP & Intensity & LBP \\
\hline 2 & 56.4 & 19.4 & 67.6 & 13.0 \\
\hline 3 & 55.7 & 17.8 & 64.2 & 14.2 \\
\hline 5 & 62.6 & 11.7 & 57.2 & 8.2 \\
\hline 8 & $\mathbf{3 1 . 8}$ & 7.5 & $\mathbf{3 4 . 9}$ & $\mathbf{0 . 2}$ \\
\hline 10 & 40.1 & 8.6 & 53.5 & 1.5 \\
\hline 12 & 35.2 & $\mathbf{6 . 4}$ & 39.5 & 1.7 \\
\hline 15 & 45.1 & 20.0 & 29.8 & 1.3 \\
\hline
\end{tabular}

\subsubsection{Volume Classification}

A test SD-OCT volume is classified as normal or abnormal based on the number of detected abnormal B-scans. Ideally, a normal SD-OCT volume should contain no abnormal B-scan, while an abnormal one should contain many. So, we consider a test volume as abnormal if it contains a certain number of detected abnormal B-scans. This threshold is taken to be equal to the average number of B-scans detected as outliers during the validation phase of the training.

For comparison, we also implemented the method proposed by Venhuizen et al. ${ }^{7}$ which used a Bag-of-Words (BoW) approach for SD-OCT volume classification. The performances of the methods are evaluated in terms of sensitivity and specificity values, and are shown in Table 2.

Table 2: Comparison of the proposed method with the Bag of Words approach. ${ }^{7}$

\begin{tabular}{|c|c|c|c|c|}
\hline Intensity & \multicolumn{2}{|c|}{ Proposed Method } & \multicolumn{2}{c|}{ BoW approach } \\
\cline { 2 - 5 } feature & SERI dataset & Duke dataset & SERI dataset & Duke dataset \\
\hline Sensitivity & $\mathbf{9 3 . 7 5}$ & 80.0 & 61.53 & 71.42 \\
Specificity & 80.0 & $\mathbf{1 0 0}$ & 58.82 & 68.75 \\
\hline
\end{tabular}

We can observe that the proposed method achieves better performances than the other method using both datasets. In particular, for SERI dataset, the proposed method achieves a sensitivity and a specificity of $93.75 \%$ and $80 \%$, while the method of Venhuizen et al. ${ }^{7}$ achieves a sensitivity and a specificity of $61.53 \%$ and $58.82 \%$. Note that these results are obtained with intensity features only, and we would expect better performance when applying the proposed methodology with texture features. The same observations apply to the results obtained with Duke dataset.

\subsubsection{Abnormal B-Scan Identification}

As mentioned previously, one main advantage of the proposed approach is the ability to identify abnormal Bscans inside a volume. This is useful, since it avoids a visual inspection of all B-scans in the volume. Furthermore, once abnormal B-scans are detected, one can focus on the segmentation of specific features such as exudates or cysts in that B-scan. Our approach differs from previous works which require a training set with manually anotated normal and abnormal B-scans, which is a tedious and time consuming task. Figure 3 shows some of the abnormal B-scans detected by the proposed method. As can be seen, B-scans with strong DME features are identified by our approach.

\section{CONCLUSION AND FUTURE WORK}

In this work we have presented a novel method for classifying SD-OCT volumes as DME or normal. As the method is based on anomaly detection, we were able to identify the individual B-scan as being diseased or not. Additionally, the method is more advantageous as we can localize the presence of disease in the entire volume. Visualization of the B-scans identified as DME indeed proved to be the diseased ones with significant DME features visible in those. Moreover, the accuracy of classification by the method shows that it outperforms Bag of Words for DME detection. As a future work, we wish to expand the model to classify more diseases of the eye like Age-related Macular Disease (AMD) along with the two classes currently considered. We also wish to gather more data and improve the accuracy of the proposed method. 

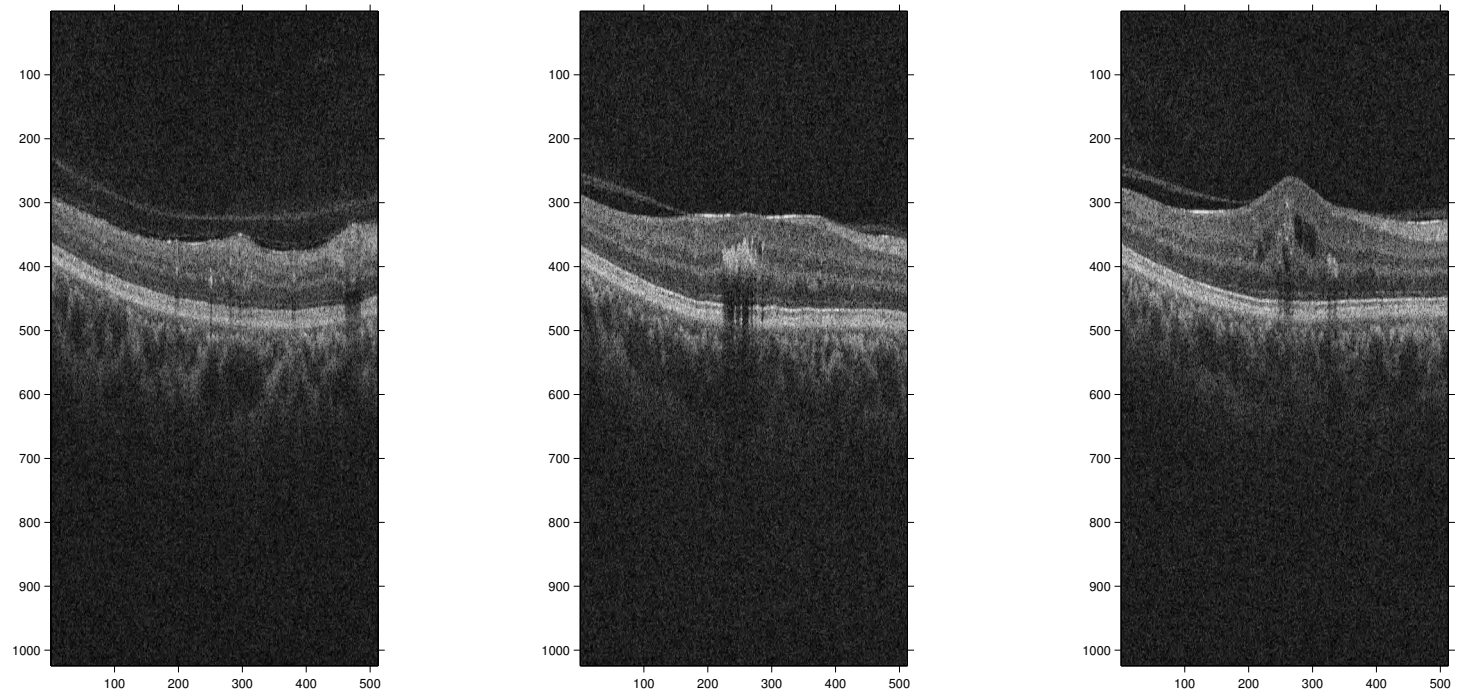

Figure 3: Some B-scans identified as diseased from a single volume classified as diseased.

\section{Aknowledgement}

This work was supported by Institut Français de Singapour (IFS) and Singapore Eye Research Institute (SERI) through the PHC Merlion program (2015-2016).

\section{REFERENCES}

[1] Abràmoff, M., Garvin, M., and Sonka, M., "Retinal imaging and image analysis," Biomedical Engineering, IEEE Reviews in 3, 169-208 (2010).

[2] Schmitt, J., Xiang, S., and Yung, K., "Speckle in optical coherence tomography," Journal of Biomedical Optics 4(1), 95-105 (1999).

[3] Buades, A., Coll, B., and Morel, J.-M., "A non-local algorithm for image denoising," in [Computer Vision and Pattern Recognition, 2005. CVPR 2005. IEEE Computer Society Conference on], 2, 60-65, IEEE (2005).

[4] Liu, Y.-Y., Chen, M., Ishikawa, H., Wollstein, G., Schuman, J. S., and Rehg, J. M., "Automated macular pathology diagnosis in retinal oct images using multi-scale spatial pyramid and local binary patterns in texture and shape encoding," Medical image analysis 15(5), 748-759 (2011).

[5] Murphy, K., [Machine learning: a probabilistic perspective], MIT Press, Cambridge, MA. (2012).

[6] Srinivasan, P., Kim, L., Mettu, P., Cousins, S., Comer, G., Izatt, J., and Farsiu, S., "Fully automated detection of diabetic macular edema and dry age-related macular degeneration from optical coherence tomography images," Biomedical Optical Express 5(10), 3568-3577 (2014).

[7] Venhuizen, F. G., van Ginneken, B., Bloemen, B., van Grinsven, M. J., Philipsen, R., Hoyng, C., Theelen, T., and Sánchez, C. I., "Automated age-related macular degeneration classification in oct using unsupervised feature learning," in [SPIE Medical Imaging], 94141I-94141I, International Society for Optics and Photonics (2015). 